\title{
The role of exercise echocardiography in the management of mitral valve disease
}

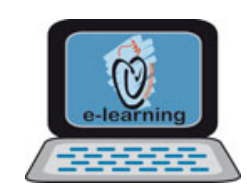

\author{
R. Jansen • P. A. M. Kracht • M. J. Cramer • \\ W. J. Tietge • L. A. van Herwerden • R. J. M. Klautz • \\ J. Kluin • S. A. J. Chamuleau
}

Published online: 20 August 2013

(C) The Author(s) 2013. This article is published with open access at Springerlink.com

\begin{abstract}
Purpose Exercise echocardiography can assess the dynamic component of mitral valve (MV) disease and may therefore be helpful for the clinical decision-making by the heart team. The purpose of this study is to determine the role of exercise echocardiography in the management of disproportionately symptomatic or otherwise atypical patients with mitral regurgitation (MR) and stenosis (MS) in clinical practice.

Methods Data of 14 MR and 14 MS patients, including echocardiograms at rest, were presented retrospectively to an experienced heart team to determine treatment strategy. Subsequently, exercise echo data were provided whereupon once again the treatment strategy was determined. This resulted in: value of exercise echo by means of 1) alteration or 2) confirmation of treatment strategy or 3) no additional value.

Results During exercise the echocardiographic severity of MV disease increased in $9(64 \%)$ MR and $8(57 \%)$ MS patients. Based upon alteration or confirmation of the treatment strategy, the value of exercise echocardiography in the management of MR and MS was $86 \%$ and $57 \%$, respectively.
\end{abstract}

The questions can be answered after the article has been published in print. You have to $\log$ in to: www.cvoi.nl.

R. Jansen · P. A. M. Kracht • M. J. Cramer •

S. A. J. Chamuleau $(\bowtie)$

Department of Cardiology, University Medical Center Utrecht,

HP E03.511, Heidelberglaan 100, 3584 CX Utrecht,

the Netherlands

e-mail: s.a.j.chamuleau@umcutrecht.nl

W. J. Tietge

Department of Cardiology, Diaconessenhuis Leiden, Houtlaan 55, 2334 CK Leiden, the Netherlands

L. A. van Herwerden · J. Kluin

Department of Cardiothoracic Surgery, University Medical Center Utrecht, Heidelberglaan 100, 3584 CX Utrecht, the Netherlands

R. J. M. Klautz

Department of Cardiothoracic Surgery, Leiden University Medical Center, Albinusdreef 2, 2333 ZA Leiden, the Netherlands
Conclusion This study showed that physical exercise echo can have an important role in the clinical decision-making of challenging patients with MV disease. Exercise echocardiography had additional value to the treatment strategy in $71 \%$ of these patients.

Keywords Exercise echocardiography $\cdot$ Physical exercise echocardiography $\cdot$ Mitral valve disease $\cdot$ Mitral regurgitation $\cdot$ Mitral stenosis $\cdot$ Clinical practice

\section{Introduction}

Mitral valve (MV) disease is a dynamic entity. Consequently, patients can present as being asymptomatic or disproportionately symptomatic in relation to the degree of severity of their valve disease on the resting echo [1]. Management of MV disease can therefore be challenging.

\section{Exercise echocardiography}

Echocardiography has been the most important and costeffective diagnostic imaging tool in clinical cardiology for more than 30 years [2]. Rest echocardiography is nowadays recommended in the diagnostic work-up of MV disease [3, 4] and has also shown to be of additional value in other valve diseases [5]. Further echocardiographic development has created new opportunities among which stress echocardiography, performed by exercise or dobutamine. Exercise echo is already widely accepted as an important diagnostic and prognostic tool in the assessment of coronary artery disease [1]. Exercise echocardiography is now also increasingly being performed in the evaluation of MV disease and has a place in the current European Society of Cardiology (ESC) guidelines. Compared with dobutamine stress echo, the main advantage of exercise echo is the direct evaluation of symptoms, exercise capacity and haemodynamic consequences of MV disease. In mitral stenosis (MS), dobutamine stress 
echo is an alternative when physical exercise cannot be performed (except for the evaluation of pulmonary pressure) [6]. However, in mitral regurgitation (MR) it is not useful due to its reduction in afterload and beneficial effect on the MR [6, 7].

As exercise echocardiography can assess the dynamic component of MR or MS, it may be helpful for clinical decision-making.

Exercise echo in mitral regurgitation

MR is the second most frequent valve disease in Western countries after aortic stenosis [8]. It can be classified as organic or functional. Two-dimensional (2D) echocardiography is the recommended method to confirm MR and must include an assessment of severity, mechanism, reparability and clinical consequences [3].

Exercise echocardiography is useful in experienced hands to quantify exercise-induced changes in organic MR, systolic pulmonary artery pressure (SPAP) and LV function [3]. The influence of exercise echo testing is evidenced by the ESC recommendations: surgery may be considered in asymptomatic patients with severe organic MR, preserved LV function and SPAP $\geq 60 \mathrm{mmHg}$ during exercise (class IIb, level of evidence C) [3]. However, a prospective trial to clarify the best treatment option in these patients started in 2013 (Dutch AMR trial) [9].

In patients with moderate functional MR, development of exercise-induced dyspnoea and increased MR severity, associated with pulmonary hypertension, are further incentives to indicate surgery (class IIa, level of evidence C) [3]. Nevertheless due to the low level of evidence, the exact value of exercise echo in the management of MR remains unclear.

\section{Exercise echo in mitral stenosis}

With the reduction in rheumatic fever, MS is now less frequently seen in industrialised countries. However, MS results in significant morbidity and mortality rates worldwide. Echocardiography is the main diagnostic method to assess severity, consequences and the extent of anatomic lesions. MS prognosis dramatically worsens with the development of symptoms [10]. Following the ESC guidelines, exercise testing is indicated in asymptomatic patients or patients with symptoms equivocal or discordant with the MS severity [3]. When analysing exerciseinduced changes in symptoms, SPAP and valve gradients may provide extra information $[3,11]$. Moreover, following the American College of Cardiology/American Heart Association (ACC/AHA) guidelines, further (invasive) intervention may be considered in (even mild) symptomatic patients, with a significant increase in SPAP $>60 \mathrm{mmHg}$ or a rise in valve gradient to $>15 \mathrm{mmHg}$ during exercise (class IIB, level of evidence C) [4]. Furthermore percutaneous mitral balloon valvotomy is indicated in asymptomatic patients with moderate to severe
MS and favourable characteristics, who reach an SPAP $>50 \mathrm{mmHg}$ at rest or $>60 \mathrm{mmHg}$ during exercise (in the absence of a left atrium thrombus or moderate to severe MR) (class I, level of evidence C) [4].

Exercise echocardiography is now increasingly being performed according to the guidelines and beyond: i.e. in disproportionately symptomatic patients in relation to their MV disease severity. The present study analysed the role of physical exercise echocardiography for the evaluation and management of disproportionately symptomatic or otherwise atypical patients with MV disease.

\section{Materials and methods}

\section{Study population}

We retrospectively evaluated 28 resting and corresponding exercise echocardiograms performed for the evaluation of MV disease in the University Medical Center of Utrecht (2009-2011).

\section{Expert panel}

Patient records were reviewed by an experienced heart team consisting of a cardiologist and cardiothoracic surgeon. The expert panel was provided with all available patient data including resting echocardiogram, whereupon they determined if intervention should be considered. Subsequently, exercise echo data were provided after which the expert panel once more decided if they would perform an intervention or watchful waiting strategy. Based on the preferred treatment strategies, depending on resting and exercise echo data respectively, three different outcomes were scored: value of exercise echo by means of either 1) change or 2) confirmation of the treatment strategy or 3) no additional value. Finally a change and/or confirmation determined the true additional value of exercise echocardiography (Fig. 1).

\section{Exercise echo protocol}

The exercise protocol was tailored to the capacity of the patient. Hence, 16 patients underwent exercise testing on a semi-supine bicycle (Fig. 2). In 12 patients alternative exercise testing was done, using a stepper or by performing knee bends. ECG and blood pressure (non-invasively) were measured before, every 3 min during and after exercise. Symptoms were continuously monitored. An adjusted protocol was used starting with a mean resistance of $30( \pm 12)$ Watts, which the physician manually increased by $10-25$ Watts every 2-3 min. Exercise testing was performed until $>85 \%$ of the age-predicted maximal heart rate was reached or when exhaustion or recognisable symptoms occurred. 
Fig. 1 Study methods: the decision flowchart per patient, used by the expert panel (1 to 5)

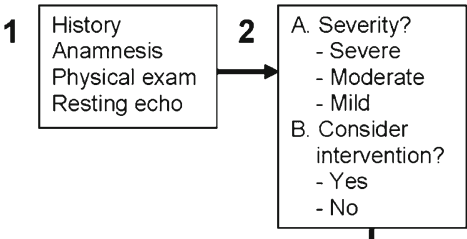

5 Comparing 2B \& 4B - Change strategy - Confirm strategy - No additional value
All patients underwent 2D transthoracic echocardiography (TTE) during physical exercise on a semi-supine bicycle or in the left lateral decubitus position directly after exercise. MR mechanism and severity were classified both on colour Doppler and according to the recommendations for quality echocardiography laboratory operations [12]. Measurement of tricuspid regurgitation (TR) jet velocity was performed in the parasternal short-axis or in the apical four-chamber view, resulting in right ventricular systolic pressure (RVSP) by adding the right atrial pressure (RAP) to the peak TR gradient. The mean transmitral gradient was measured by tracing the area-under-the-curve of the mitral $\mathrm{E}$ and $\mathrm{A}$ waves obtained by continuous Doppler in the apical views.

Examinations were performed with the Philips 5500 or Philips IE33 (Philips Medical Systems, Andover, Massachusetts, USA) echo machines. All datasets were archived on the hospital server as video loops and freeze frames in a digital format (DICOM). Off-line analysis was performed using Xcelera software.

\section{Results}

Mitral regurgitation

\section{Patient characteristics}

The MR group consisted of 14 patients (7 organic and 7 functional MR). Mean age was 66 (31-78) years. All patients

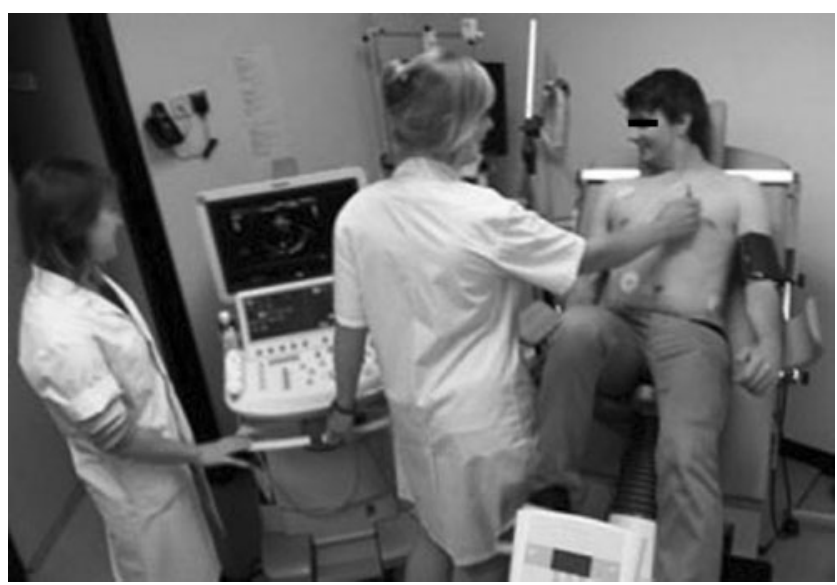

Fig. 2 Exercise echocardiography on a semi-supine bicycle in the University Medical Center of Utrecht were symptomatic. The LV function was normal in 11 patients $(79 \%)$.

MR severity at rest was classified at baseline: 1 severe, 4 moderate and 7 mild. Two patients had clinical suspicion of MR but no regurgitation on resting images (Tables 1 and 2).

\section{Exercise echo}

The mean heart rate during exercise was $131(80-160)$ beats per minute (bpm). Nine patients $(64 \%)$ reached $>85 \%$ of the age-predicted maximal heart rate (mean $145 \pm 12 \mathrm{bpm}$ ). In $43 \% \beta$-adrenergic blocking medication was used. Severity of MR during exercise increased in 9/14 patients (64\%). During exercise, 4 patients had severe MR. Exercise RVSP was $\geq 60 \mathrm{mmHg}$ in $5(36 \%)$ subjects, of whom $3(60 \%)$ were diagnosed with severe MR during exercise (Table 2). In 10 patients $(71 \%)$ stress RVSP was higher compared with the resting value ( 5 patients $\geq 60 \mathrm{mmHg}$ ), and in 4 subjects a change in RVSP could not be determined. Figure 3a shows the increase in mean RVSP from $29.9 \pm 4.5 \mathrm{mmHg}$ at rest to $54.2 \pm 15.8 \mathrm{mmHg}$ during exercise.

\section{Clinical management}

Based on the judgment of the expert panel an intervention was indicated in $5 \mathrm{MR}$ patients (80\% new indication); 4 patients in this group had severe MR during exercise (of whom 1 already had severe MR at rest) and in 4 patients exercise RVSP was $\geq 60 \mathrm{mmHg}$ (Table 2). In 1 patient with RVSP $\geq 60 \mathrm{mmHg}$ during exercise but at the same time moderate MR, an intervention was not recommended.

Figure $4 \mathrm{a}$ demonstrates a change in recommended treatment strategy for MR patients in $29 \%$ and confirmation in $57 \%$. The value of physical exercise echocardiography in the management of MR was demonstrated in $86 \%$.

Mitral stenosis

\section{Patient characteristics}

The MS group consisted of 14 patients of whom 7 (50\%) had organic disease and 7 (50\%) had MS after mitral valve repair (MVR). Mean age was 56 (29-83) years and $86 \%$ were symptomatic. LV function was normal in $93 \%$. Severity of MS at rest was classified at baseline: 1 severe, 11 moderate and 2 mild (Tables 1 and 3 ). 
Table 1 Baseline characteristics

\begin{tabular}{lll}
\hline Baseline & MR & MS \\
\hline $\begin{array}{l}\text { Patient characteristics } \\
\text { Study group }\end{array}$ & 14 & 14 \\
Age (range) & $66(31-78)$ & $56(29-83)$ \\
Gender: & & \\
Male (\%) & 50 & 29 \\
Female (\%) & 50 & 71 \\
Left ventricular function & & \\
Normal & 11 & 13 \\
Dysfunctional & \\
Mitral valve disease & 3 & 1 \\
Symptomatic & & \\
Severity in rest: & 14 & 12 \\
Severe & 1 & 1 \\
Moderate & 4 & 11 \\
Mild & 7 & 2 \\
None & 2 & 0 \\
Exercise protocol & 10 & 6 \\
Semi-supine bicycle & 4 & \\
Alternative exercise test & & \\
\hline
\end{tabular}

$M R$ mitral regurgitation, $M S$ mitral stenosis

${ }^{a}$ According to the ESC guidelines: left ventricular ejection fraction of $\leq 60 \%$ or left ventricular end systolic dimension of $\geq 45 \mathrm{~mm} \mathrm{[4]}$

${ }^{\mathrm{b}}$ Using a stepper or performing knee bends

\section{Exercise echo}

During exercise echocardiography the mean heart rate was $115(80-150) \mathrm{bpm}$. Four patients (29\%) achieved $>85 \%$ of the age-predicted maximal heart rate (mean $143 \pm 9 \mathrm{bpm}$ ). Of these patients $71 \%$ used $\beta$-adrenergic blocking medication. MS severity during exercise increased in $8 / 14$ patients $(57 \%)$. Seven patients had severe MS during exercise. Exercise-induced RVSP was $\geq 60 \mathrm{mmHg}$ in 4 (29\%) of the MS patients. Three of them (75\%) were diagnosed with severe MS during exercise. Stress mean gradient values increased to $>15 \mathrm{mmHg}$ in 7 (50\%) patients. All were diagnosed with severe MS during exercise (Table 3).

RVSP increased in 11 patients (79\%) during exercise (4 patients $\geq 60 \mathrm{mmHg}$ ), whereas in 2 cases RVSP could not be determined. One patient showed a slight decrease in RVSP. However, with severe MS and exercise-induced mean gradient $>15 \mathrm{mmHg}$, intervention was still recommended in this patient by the expert panel.

Figure $3 \mathrm{~b}$ and $\mathrm{c}$ show a rise in mean RVSP and mean gradient during exercise from $34.9 \pm 9.0 \mathrm{mmHg}$ to $52.1 \pm 17.8 \mathrm{mmHg}$ and from $6.9 \pm 2.4 \mathrm{mmHg}$ to $15.9 \pm 7.3 \mathrm{mmHg}$, respectively.

\section{Clinical management}

The expert panel recommended an intervention in every patient who developed severe MS during exercise (7). For 6 patients this indication was new (Table 3 ). In all 7 subjects exercise-induced mean gradient was $>15 \mathrm{mmHg}$ and 3 (43\%) had an RVSP $\geq 60 \mathrm{mmHg}$. Of the patients with exercise RVSP $\geq 60 \mathrm{mmHg}, 75 \%$ received an indication for surgery.

Figure $4 \mathrm{~b}$ shows a change in recommended treatment strategy in $43 \%$ of all MS patients and confirmation in $14 \%$. The value of physical exercise echocardiography in the management of MS was seen in $57 \%$.

\section{Discussion}

The present study is remarkable for its objective to define the clinical value of exercise echocardiography for decisionmaking in challenging patients with MS or MR who cannot easily be classified by the guidelines.

As clearly described by Van de Heyning et al. [13], several articles have already pointed out the increasing evidence for exercise echo in determining dynamic changes in MV disease and to identify high-risk patients who may benefit from (early) intervention. In addition, our study showed that exercise echocardiography was of additional value in the clinical decision-making in 20/28 (71\%) of all patients, demonstrating 10 patients with a change and 10 patients with confirmation of treatment strategy.

\section{Exercise-induced changes in MV disease severity}

Data supporting exercise-induced changes in echocardiographic parameters to quantify $\mathrm{MR}$ are limited [3, 11]. Only an exercise-induced increase of the effective regurgitant orifice area (EROA) by $\geq 13 \mathrm{~mm}$ has shown to be associated with a significant increase in relative risk of death and hospitalisation for cardiac decompensation in functional MR [14]. Unfortunately EROA is difficult to measure, especially during exercise. Furthermore, exercise echocardiography has only shown to be useful in observing symptoms caused by an increase in MR severity in symptomatic patients with mild rheumatic MR at rest [15].

In our study MS severity was mainly based on the mean gradient value. Determining MS severity by mean gradient using exercise echocardiography has proven to be feasible and well correlated with invasive measurements [16-18]. Previous studies also demonstrated marked elevations in mean gradient after peak exercise in patients with predominantly MS [19-23]. Further (invasive) intervention may therefore be considered in (even mild) symptomatic patients with a significant increase in valve gradient to $>15 \mathrm{mmHg}$ during exercise (class IIB, level of evidence C) [4]. 


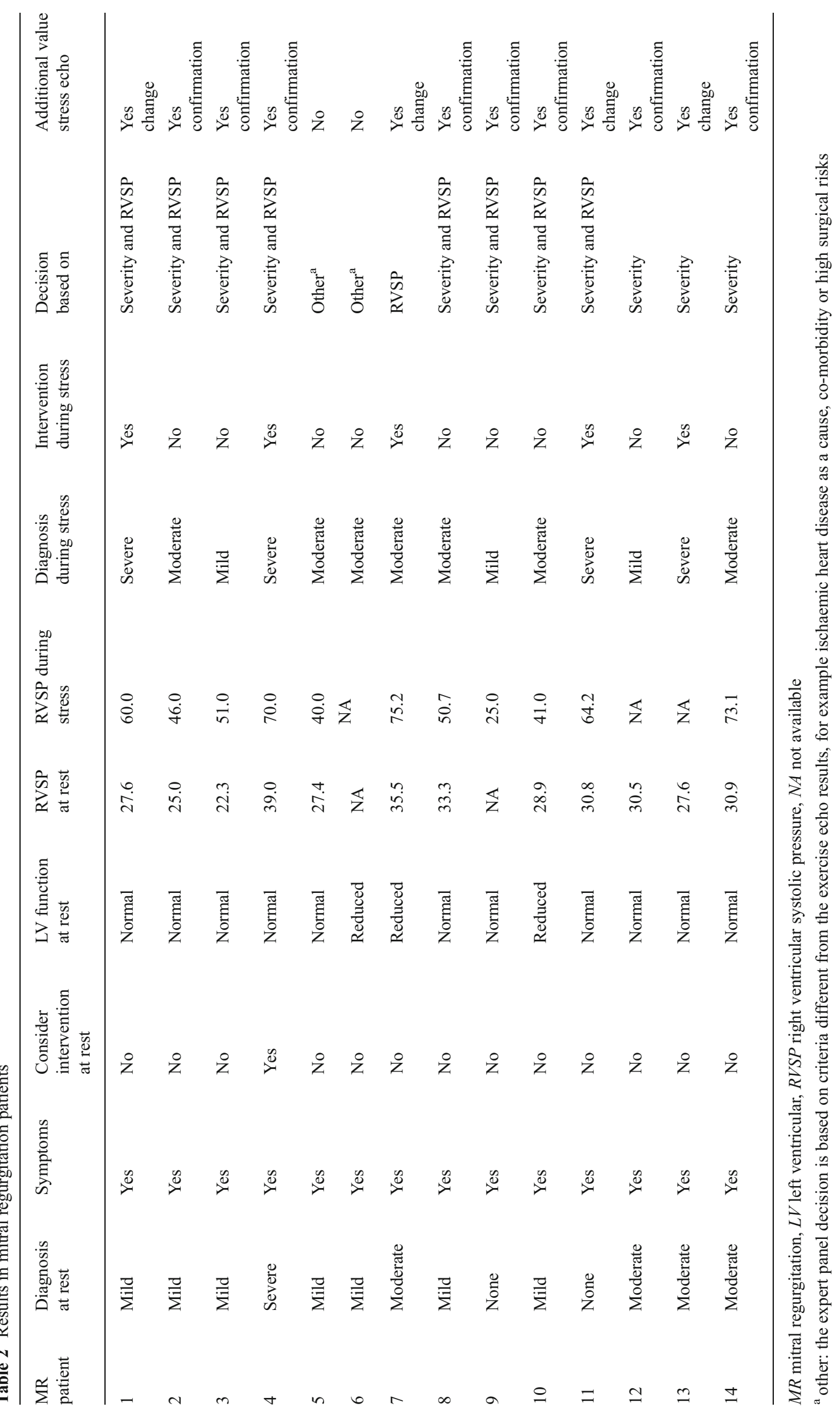


a

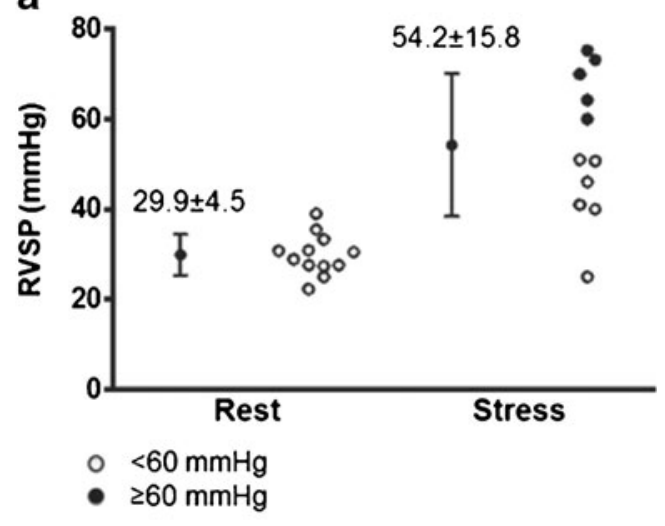

b

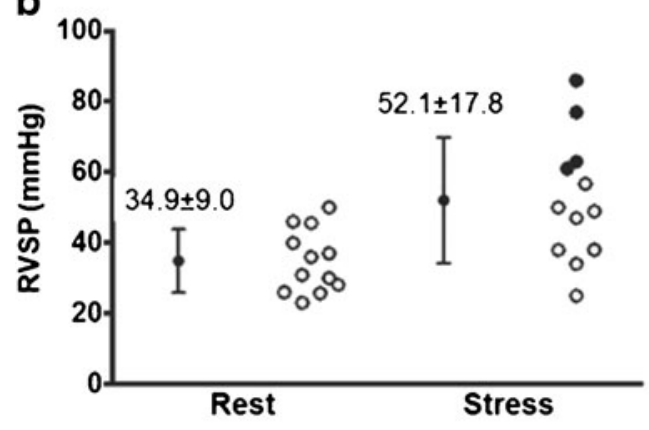

- $<60 \mathrm{mmHg}$

- $260 \mathrm{mmHg}$

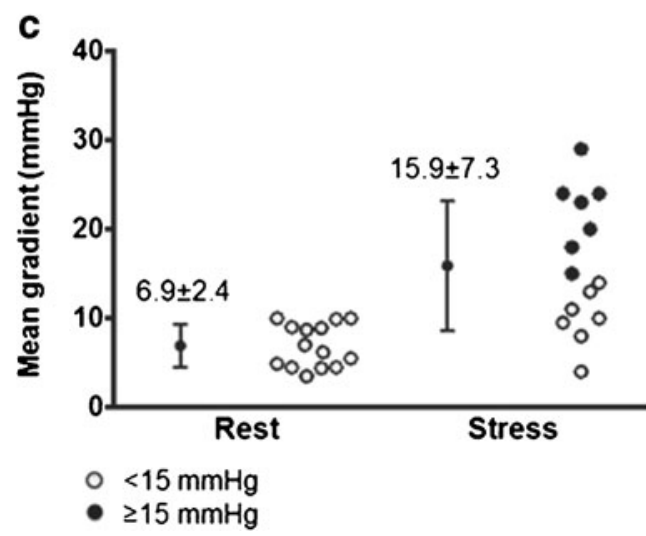

Fig. 3 Right ventricular systolic pressure (RVSP) values in patients with mitral regurgitation (a) and mitral stenosis (b) and mean gradient values in mitral stenosis (c) during rest and exercise echocardiography

As a result, in all $7 \mathrm{MS}$ subjects with an exercise-induced mean gradient $>15 \mathrm{mmHg}$, our expert panel recommended an intervention. However, the proposed cut-off value by the guidelines $(>15 \mathrm{mmHg})$ is arbitrary, consensus driven and not supported by solid evidence.

Exercise-induced changes in pulmonary pressures

Echocardiography has been validated as the principal tool for measuring pulmonary pressures in daily practice, although a

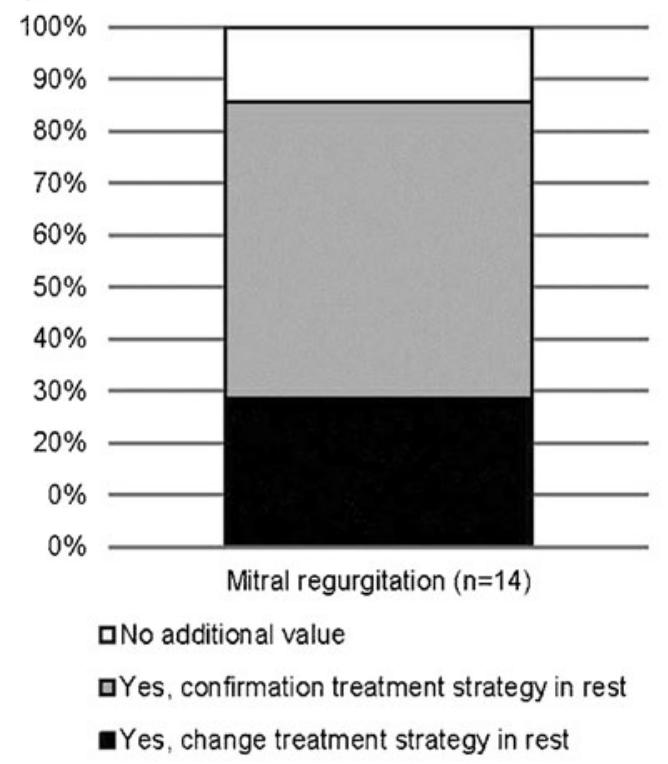

b

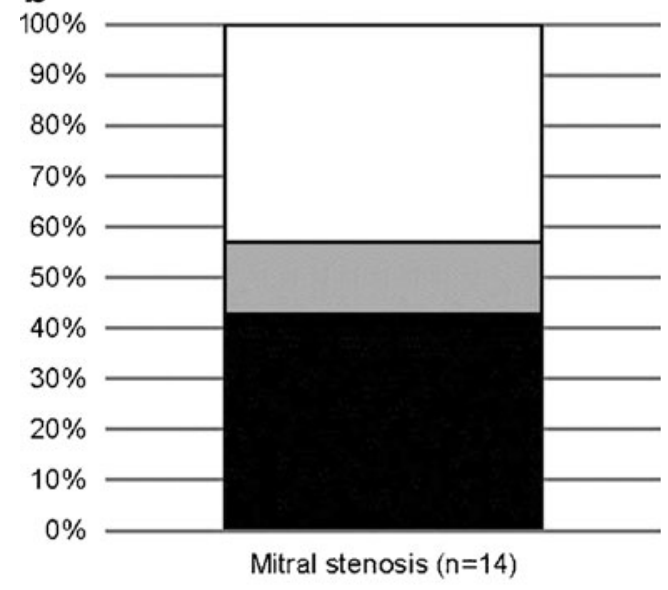

口No additional value

口Yes, confirmation treatment strategy in rest

-Yes, change treatment strategy in rest

Fig. 4 The value of exercise echocardiography in mitral regurgitation (a) and mitral stenosis (b)

discrepancy exists on the correlation between echocardiographic findings and invasive measurements [24]. Guidelines regarding MS urge to consider further intervention in symptomatic patients with significant elevation of SPAP (>60 $\mathrm{mmHg}$ ) during exercise $[19,25,26]$. Furthermore, marked pulmonary hypertension (SPAP $>50 \mathrm{mmHg}$ at rest or $>60 \mathrm{mmHg}$ during exercise) is associated with a poor prognosis in patients with LV dysfunction [27]. Despite a low level of evidence, echocardiographic measurement of an exercise-induced SPAP $\geq 60 \mathrm{mmHg}$ has therefore also been suggested as cut-off value for considering MVR in asymptomatic patients with severe organic MR and preserved LV 


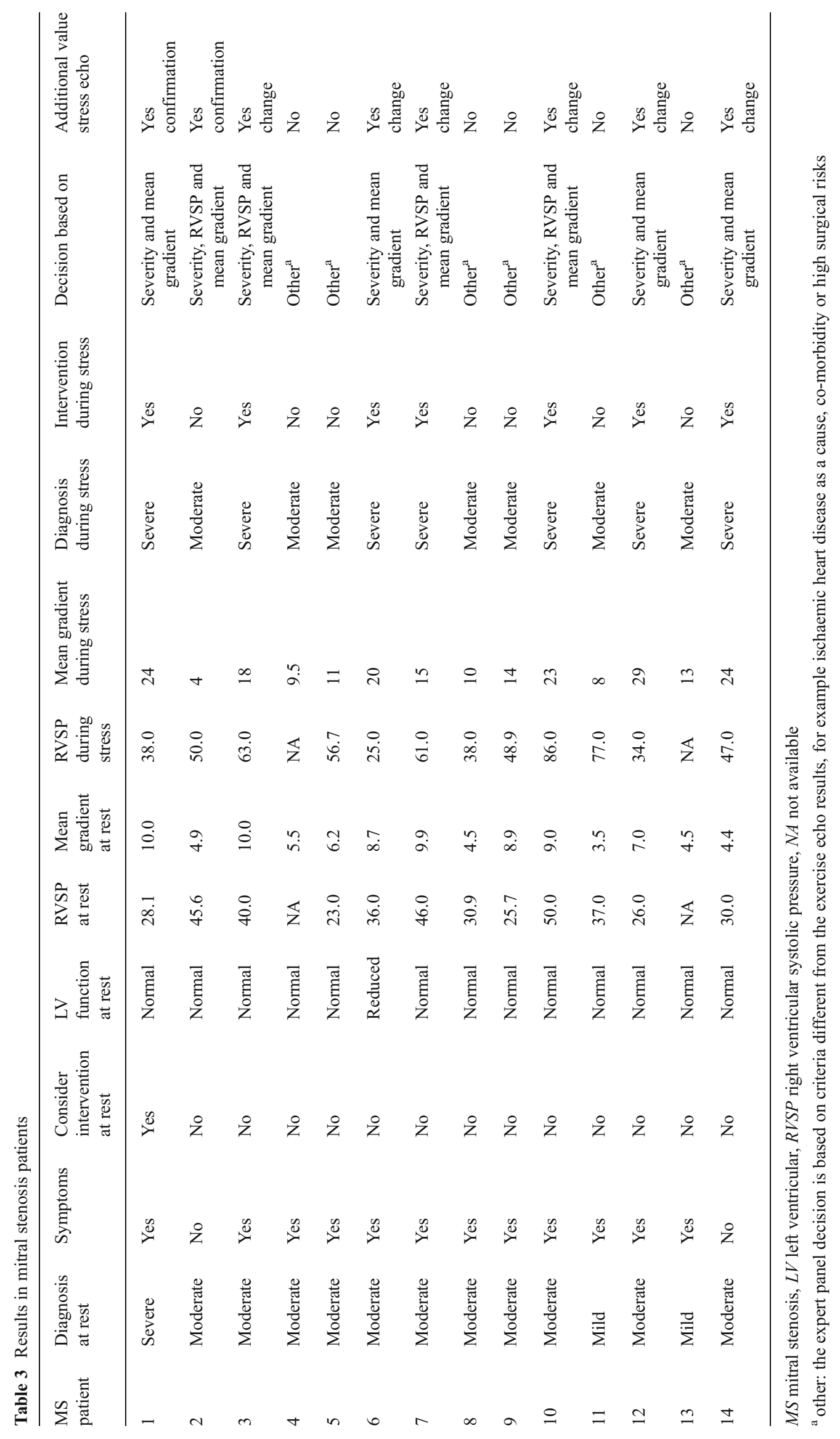


function [3]. Moreover, Lebrun et al. showed that SPAP values were greatest in patients with marked dynamic MR, and well correlated with increases in regurgitant volume (PISA method) [28].

However, no reference values exist for physiological exercise-induced changes in SPAP. Also other factors than MV disease can play a role in the dynamic changes during exercise, e.g. alterations of pulmonary resistance and neurohormonal activation [28].

In absence of right-sided flow obstruction, SPAP and RVSP values were equal in this study. Although stress RVSP peak values of $35-45 \mathrm{mmHg}$ are regarded as physiological [29-31] in (young, male) endurance athletes and older patients ( $\geq 60$ years), peak-exercise values of $>60 \mathrm{mmHg}$ have been reported $[32,33]$. The prognostic significance of this marked increase has not been defined. It has been advocated to integrate age and level of exercise with the exerciseinduced SPAP response to determine abnormal elevation of pulmonary pressures [24, 29, 33]. In our study a stress SPAP of $\geq 60 \mathrm{mmHg}$ in patients with less than maximal workload was classified as pathological.

The expert panel's decision was influenced by the RVSP measurement during exercise in 13 (46\%) of all patients. In 15 patients management was determined based on other criteria, e.g. an increase in mean gradient (an intervention was recommended in all MS patients with mean gradient $>15 \mathrm{mmHg}$ ).

\section{Value of exercise echocardiography}

For optimal exercise testing, a heart rate of $>85 \%$ of the agepredicted maximal heart rate was obligatory. In $64 \%$ of the MR patients this criteria was achieved, versus only $29 \%$ of the MS patients. Moreover, the total mean heart rate appeared to be lower in MS compared with MR (115 versus $131 \mathrm{bpm})$. Both remarks can be explained by the use of more $\beta$-adrenergic blocking agents in the MS population compared with MR patients ( $71 \%$ versus $43 \%$ ).

Although exercise echocardiography is only recommended in asymptomatic patients with severe MR [3], in this study we analysed symptomatic MR patients. Apparently, in daily clinical practice exercise echocardiography is more and more supposed to be of additional value in disproportionately symptomatic patients. Furthermore, the additional value in 12 symptomatic ( $86 \%$ ) and 2 asymptomatic (14\%) MS patients certainly fits within the earlier described guideline recommendations for exercise echocardiography in both asymptomatic and symptomatic MS [3, 4].

\section{Limitations}

This study was retrospective with its inherent limitations. The decision to perform an exercise echocardiography had already been made by the physician, thus our patient population is a selected one. Because patient numbers were rather small, no subanalysis for specific MR or MS aetiologies could be performed. We also did not focus on the exact difference in LV function by measuring ejection fraction at rest and during exercise, but in our patient cohort it appeared to be less of importance. Finally, the expert panel judgment did not account for the interaction between patient and treating physician as this is not possible in this type of research. This might ultimately have resulted in a different treatment strategy by the expert panel compared with the treating physician.

Only 2 patients were asymptomatic at rest. Evaluation of symptomatic patients remains subjective and is solely based on the patient's history and cardiologist's interpretation. Nevertheless, being symptomatic is of utmost importance in the recommended guidelines for MV surgery and therefore important in determining the best treatment strategy $[3,4]$. Thus, exercise echocardiography also serves as an important additional tool to unmask specific symptoms in atypical patients.

Concerning the technical limitations, evaluation of echocardiographic examination is sometimes limited by a poor imaging window, which is even more difficult during exercise. Therefore considerable experience is needed to obtain good quality echo studies. Moreover, patients may be unable to exercise, or perform a submaximal test. Furthermore, while exercise-related symptoms may sometimes be difficult to interpret and remain subjective, additional haemodynamic parameters during exercise are indispensable. However these (noninvasive) measurements can be difficult to perform during exercise, differ in accuracy and may not be fully established.

\section{Future prospective}

A prospective trial is needed to confirm the value of exercise echocardiography in the management of MV disease. Subanalysis of various mechanisms is necessary since they are based on different aetiologies and are diagnosed and treated accordingly.

A validated and standardised exercise protocol is necessary in order to compare exercise echo results. Exercise endpoints should be determined at $>85 \%$ of the age-predicted maximum heart rate, or the occurrence of recognisable symptoms.

Furthermore, there may be an important role for real-time three dimensional (3D) echocardiography as an alternative for 2D exercise echocardiography [34].

\section{Conclusion}

Exercise echocardiography is an important tool to unravel dynamic changes and monitor symptomatic responses occurring in 
MV disease during physical exercise. It can support the heart team in determining the best treatment strategy in disproportionately symptomatic or otherwise atypical patients, as seen in $71 \%$ of our study patients. A prospective trial is needed to support our findings by confirming the additional value of exercise echocardiography in patients with MV disease and its different aetiologies.

\section{Funding None}

\section{Conflicts of interest None declared.}

Open Access This article is distributed under the terms of the Creative Commons Attribution License which permits any use, distribution, and reproduction in any medium, provided the original author(s) and the source are credited.

\section{References}

1. Lancellotti P, Magne J. Stress testing for the evaluation of patients with mitral regurgitation. Curr Opin Cardiol. 2012;27:492-8.

2. Kamp O, Cramer MJM. History of echocardiography in the Netherlands: 30 years of education and clinical applications. Neth Heart J. 2008;16:16-20.

3. Vahanian A, Alfieri O, Andreotti F, et al. Guidelines on the management of valvular heart disease (version 2012): The Joint Task Force on the Management of Valvular Heart Disease of the European Society of Cardiology (ESC) and the European Association for Cardio-Thoracic Surgery (EACTS). Eur J Cardiothorac Surg. 2012;42:S1-S44.

4. Bonow RO, Carabello BA, Chatterjee K, et al. ACC/AHA 2006 guidelines for the management of patients with valvular heart disease: a report of the American College of Cardiology/American Heart Association Task Force on Practice Guidelines (Writing committee to revise the 1998 guidelines for the management of patients with Valvular Heart Disease). Circulation. 2006;114:e84-e231.

5. Joziasse IC, Vink A, Cramer MJ, et al. Biscupid stenotic aortic valves: clinical characteristics and morphological assessment using MRI and echocardiography. Neth Heart J. 2011;19:119-25.

6. Piérard A, Lancellotti P. Stress testing in valve disease. Heart. 2007;93:766-72.

7. Heinle SK, Tice FD, Kisslo J. Effect of dobutamine stress echocardiography on mitral regurgitation. J Am Coll Cardiol. 1995;25: 122-7.

8. Iung B, Baron G, Butchart EG, et al. A prospective survey of patients with valvular heart disease in Europe: The Euro Heart Survey on Valvular Heart Disease. Eur Heart J. 2003;24:1231-43.

9. Tietge WJ, de Heer LM, van Hessen MW, et al. Early mitral valve repair versus watchful waiting in patients with severe asymptomatic organic mitral regurgitation; rationale and design of the Dutch AMR trial, a multicenter, randomised trial. Neth Heart J. 2012;20:94-101.

10. Horstkotte D, Niehues R, Strauer BE. Pathomorphological aspects, aetiology and natural history of acquired mitral valve stenosis. Eur Heart J. 1991;12(Suppl B):55.

11. Picano E, Pibarot $P$, Lancellotti $P$, et al. The emerging role of exercise testing and stress echocardiography in valvular heart disease. J Am Coll Cardiol. 2009;54:2251-60.

12. Picard MH, Adams D, Bierig SM, et al. American Society of Echocardiography recommendations for quality echocardiography laboratory operations. J Am Soc Echocardiogr. 2011;24:1-10.
13. Van de Heyning CM, Magne J, Lancellotti P, et al. The importance of exercise echocardiography for clinical decision making in primary mitral regurgitation. J Cardiovasc Med. 2012;13:260-5.

14. Lancellotti P, Gérard P, Piérard L. Long term outcome of patients with heart failure and dynamic functional mitral regurgitation. Eur Heart J. 2005;26:1528-32.

15. Tischler MD, Battle RW, Saha M, et al. Observations suggesting a high incidence of exercise-induced severe mitral regurgitation in patients with mild rheumatic mitral valve disease at rest. J Am Coll Cardiol. 1995;25:128-33.

16. Voelker W, Jaksch R, Dittman H, et al. Validation of continuous-wave Doppler measurements of mitral valve gradients during exercise- A simultaneous Doppler-catheter study. Eur Heart J. 1989;10:737-46.

17. Sagar KB, Wann SL, Paulson WJ, et al. Role of exercise Doppler echocardiography in isolated mitral stenosis. Chest. 1987;92:27-30.

18. Braverman AC, Thomas JD, Lee RT. Doppler echocardiographic estimation of mitral valve area during changing hemodynamic conditions. Am J Cardiol. 1991;68:1485-90.

19. Leavitt JI, Coats MH, Falk RH. Effects of exercise on transmitral gradient and pulmonary artery pressure in patients with mitral stenosis or a prosthetic mitral valve: a Doppler echocardiographic study. J Am Coll Cardiol. 1991;17:1520-6.

20. Voelker W, Berner A, Regele B, et al. Effect of exercise on valvular resistance in patients with mitral stenosis. J Am Coll Cardiol. 1993;22:777-82.

21. Tunick PA, Freedberg RS, Gargiulo A, et al. Exercise Doppler echocardiography as an aid to clinical decision making in mitral valve disease. J Am Soc Echocardiogr. 1992;5:225-30.

22. Hecker S, Zabalgoitia M, Ashline P, et al. Comparison of exercise and dobutamine stress echocardiography in assessing mitral stenosis. Am J Cardiol. 1997;80:1374-7.

23. Song JK, Kang DH, Lee CW, et al. Factors determining the exercise capacity in mitral stenosis. Am J Cardiol. 1996;78:1060-2.

24. Hoendermis ES. Pulmonary arterial hypertension: an update. Neth Heart J. 2011;19:514-22.

25. Kasalicky J, Hurych J, Widimsky J, et al. Left heart haemodynamics at rest and during exercise in patients with mitral stenosis. Br Heart $\mathrm{J}$. 1968;30:188-95.

26. Cheriex EC, Pieters FA, Janssen JH, et al. Value of exercise Doppler-echocardiography in patients with mitral stenosis. Int $\mathrm{J}$ Cardiol. 1994;45:219-26.

27. Abramson SV, Burke JF, Kelly Jr JJ, et al. Pulmonary hypertension predicts mortality and morbidity in patients with dilated cardiomyopathy. Ann Intern Med. 1992;116:888-95.

28. Lebrun F, Lancellotti P, Pierard LA. Quantitation of functional mitral regurgitation during bicycle exercise in patients with heart failure. JACC. 2001;38:1685-92.

29. Bossone E, Rubenfire M, Bach DS, et al. Range of tricuspid regurgitation velocity at rest and during exercise in normal adult men: implications for the diagnosis of pulmonary hypertension. J Am Coll Cardiol. 1999;33:1662-6.

30. Janosi A, Apor P, Hankoczy J, et al. Pulmonary artery pressure and oxygen consumption measurement during supine bicycle exercise. Chest. 1988;93:419-21.

31. Argiento P, Chesler N, Mule M, et al. Exercise stress echocardiography for the study of the pulmonary circulation. Eur Respir J. 2010;35:1273-8.

32. La Gerche A, Heidbüchel H, Burns AT, et al. Disproportionate exercise load and remodeling of the athlete's right ventricle. Med Sci Sports Exerc. 2011;43:974-81.

33. Mahjoub H, Levy F, Cassol M, et al. Effects of age on pulmonary artery systolic pressure at rest and during exercise in normal adults. Eur J Echocardiogr. 2009;10:635-40.

34. Leung KY, van Stralen M, Danilouchkine MG, et al. Automated analysis of three-dimensional stress echocardiography. Neth Heart J. 2011;19:307-10. 


\section{CVOI E-learning formula!}

This is the CVOI e-learning article. The author has prepared 10 questions which are available through the website of the Cardiovascular Educational Institute (CVOI). Please follow the instructions below.

After finishing the questions you will be asked to fill in your name, hospital and e-mail address; then press the button 'verzenden'.

When 6 out of the 10 questions are answered correctly, you acquire 1 accreditation point granted by the Quality Committee of the Netherlands Society of Cardiology (NVVC). The acquired point will be credited to your personal file in the GAIA system. You will also receive an e-mail with all the correct answers.

Over a period of one year 10 e-learning articles will appear in 10 subsequent $\mathrm{NHJ}$ editions. In each edition the e-learning article will be recognisable by a special icon. On an annual basis you can collect 10 accreditation points. The accreditation points are credited in the GAIA system by the CVOI.

If you need additional information, please contact the CVOI by e-mail: cvoi@cvoi.org or by phone: 030-2345001.

E.E. van der Wall

Chief editor NHJ
K.B. Schick

Coordinator CVOI 